\title{
Información turística de cuatro comunidades de Pedro Pablo Gómez, Jipijapa, Manabí, Ecuador
}

\section{Tourist information of four communities of Pedro Pablo Gómez, Jipijapa, Manabí, Ecuador}

\author{
Juan Francisco Villamar González ${ }^{1}$, Erika Belén Marcillo Rodríguez ${ }^{1}$, Olga María Bravo Guerrero $^{1}$ y Melanie \\ Sofía Encalada Menéndez ${ }^{1}$ \\ ${ }^{1}$ Universidad Estatal del Sur de Manabí \\ *juan.villamar@unesum.edu.ec
}

DOI: https://doi.org/10.26871/killkana_social.v3i2.467

\begin{abstract}
Resumen
La sistematización de la información permite dar a conocer y entender datos de una manera eficaz. El objetivo del trabajo consistió en recopilar información turística de las comunidades San Jacinto, La Línea, La Cabuya y La Curia de San Jacinto de Pedro Pablo Gómez, Jipijapa. Se identificaron los stakeholders y se realizaron encuestas, observación y exploración del sector turístico. Se obtuvo información ecológica, antropológica, y económica de las comunidades. Se determinó la importancia de los stakeholders, según la utilidad que representan, y el rol que cumple en el ámbito turístico, y el aporte principal en las comunidades. De esta forma se busca la socialización de la información a los representantes y habitantes de las comunidades, para que pueda ser utilizada en beneficio y permita un buen desarrollo turístico en las comunidades involucradas.
\end{abstract}

Palabras clave: Sistematización, stakeholders, comunidades, turismo.

\begin{abstract}
The Information's systematization allows knowing and understanding data in an efficient way. The objective of this investigation was to collect touristic information of the following communities San Jacinto, La Linea, La Cabuya, La Curia in Pedro Pablo Gómez, Jipijapa. In addition, stakeholders were identified and the tools used to gather data were surveys, observation and exploration of the tourism sector were conducted. Ecologic, anthropologic and economic information was obtained from the communities. The importance of the stakeholders was determined, according to the importance they represent, their role taken in tourism field, and the main contribution in the communities. In this way, it can be socialize the information to the representatives and habitants of the communities, so, this investigation can also be used by the leaders of the communities where the study was taken in order to be implemented for touristic developments of their places.
\end{abstract}

Key words: systematization, stakeholders, communities, tourism.

\section{Introducción}

Tomando como referencia a Jara, la sistematización es la interpretación crítica de una o varias experiencias que a partir de su ordenamiento y reconstrucción, descubre o explicita la lógica del proceso vivido, los factores que han intervenido en dicho proceso, cómo se han relacionado entre sí y por qué lo han hecho de ese modo. Por lo que en la actualidad, la sistematización de la información en el ámbito turístico va tomando más importancia. Esta es una clave para poder intercambiar información y dar a conocer un producto o servicio al turista.

En la provincia de Manabí existen comunidades con mucha riqueza turística, que ofrecen sus recursos y atracti- vos a turistas que no logran tener la información necesaria para sentirse atraídos, ya que el turista basa su decisión a la accesibilidad de información que tenga, lo que hace que se manifieste la necesidad de crear sistemas de información y los propios residentes puedan participar y aportar al desarrollo de estas para consolidar el turismo en la comunidad.

La aplicación del trabajo de investigación en las comunidades La Curia, La Línea, La Cabuya y San Jacinto en la Parroquia rural Pedro Pablo Gómez del cantón Jipijapa busca recaudar información sobre el área y residentes, sistematizar todos estos datos encontrados para que los turistas puedan obtener lo requerido de una forma más factible, clara y así convertirse en una fuente precisa para los usuarios. 
Teniendo en cuenta la definición postulada por Freeman en 1984, los stakeholders del turismo son "aquellas personas o grupos de personas procedentes de entidades públicas o privadas que pueden afectar o son afectadas por las actividades turísticas y que, por lo tanto, deben ser considerados como un elemento esencial en la planificación estratégica del sector del turismo en un territorio o destino turístico". Siendo esta la referencia, mediante esta misma investigación se identificarán a los stakeholders interesados en intervenir en el sector turístico, con el fin de que logren promocionar estas comunidades desde el punto de vista turistico.

\section{Metodología}

La investigación se apoyó en los siguientes métodos y técnicas para la recolección de la información.

\subsection{Método Correlacional}

De acuerdo con Briones, consiste en la búsqueda de algún tipo de relación entre dos o más variables, y en qué medida la variación de una de las variables afecta a la otra ya sea positiva o negativa, sin llegar a conocer cuál de ellas puede ser causa o efecto. La información que se recoja sobre las variables involucradas en la relación comprobará o no esa relación, en cuando a su magnitud, dirección y naturaleza

\subsection{De campo}

Se realizaron visitas a las Comunidades San Jacinto, la Línea, la Cabuya, y la Curia pertenecientes a la parro- quia Pedro Pablo Gómez para poder realizar el respectivo levantamiento de información.

\subsection{Inductivo - deductivo}

Se realizó un estudio de los hechos, objeto de la investigación partiendo de lo particular a lo general. Entrevista a los involucrados en el problema investigado.

Al realizar la visita a las comunidades, el diálogo con los moradores se identificó a los stakeholders de las comunidades, los cuales se caracterizan en un cuadro de datos, también se realizó la caracterización antropológica, Se analizó estadísticamente el comportamiento de la actividad de producción y distribución económica, se describió desde la ecología, las áreas de interés para el turismo para su posterior sistematización su relación con el desarrollo turístico.

\subsection{Técnica}

Encuestas dirigidas a los moradores de las comunidades; y habitantes de las comunidades que trabajan en las fincas de la zona. Encuestas que serán reflejadas en los resultados.

Entrevistas dirigidas a los moradores de las comunidades: dueños de fincas y presidentes de las comunidades visitadas, para identificar los stakeholders en estas comunidades.

\section{Resultados}

Tabla 1 Describir desde el punto de vista ecológico un área de interés turístico en las comunidades La Curia, La Línea, La Cabuya y San Jacinto perteneciente a la parroquia Pedro Pablo Gómez.

\begin{tabular}{|c|c|}
\hline $\begin{array}{l}\text { Descripción y valoración } \\
\text { ecológica de un área de } \\
\text { interés turístico. }\end{array}$ & $\begin{array}{l}\text { De acuerdo a los datos obtenidos, se concluye que la diversidad de la flora y la fauna de las comunidades en Pedro } \\
\text { Pablo Gómez es variada. Ya que esta, en lo que respecta a flora, cuenta con plantas alimenticias, ornamentales, entre } \\
\text { otros. Por otro lado, en la fauna existen animales domésticos. } \\
\text { De acuerdo a la observación realizada en la práctica, se pudo constatar que el sitio se mantiene en su hábitat natural } \\
\text { al no haber edificios ni contaminación, además de que cuentan con tachos de basuras en donde se mantiene el orden } \\
\text { de la limpieza. }\end{array}$ \\
\hline $\begin{array}{l}\text { Problemas ambientales } \\
\text { generados por el turismo } \\
\text { en ambientes naturales. }\end{array}$ & $\begin{array}{l}\text { Si la actividad turística se desarrollara completamente en estas comunidades impactaría de manera positiva y negativa } \\
\text { al medio ambiente, en especial por las malas prácticas de algunas de las actividades que realicen los turistas, como } \\
\text { por ejemplo: la recolección de especies de flora debido a su encanto y belleza, la fotografía, la caza a especies de } \\
\text { fauna y el mal uso del fuego pueden resultar afectando al ambiente. }\end{array}$ \\
\hline Turismo y sociedad & $\begin{array}{l}\text { En la actualidad, las comunidades involucradas en el presente trabajo, aún no muestran tener un impacto en el entorno } \\
\text { que perjudique al medio ambiente, por lo mismo que el turismo en estas comunidades es escaso, y tampoco tienen } \\
\text { una infraestructura que logre contaminar el medio. Estas personas son conscientes del cuidado que se debe tener a } \\
\text { la naturaleza, y califican a sus comunidades como sectores "humildes". }\end{array}$ \\
\hline $\begin{array}{l}\text { Espacios turísticos y su } \\
\text { impacto en la ecología. }\end{array}$ & $\begin{array}{l}\text { Medidas de prevención y mitigación para la sostenibilidad turística teniendo como referencia principal las adapta- } \\
\text { ciones y acciones de mitigación al cambio climático en las comunidades, y de cómo en la creación y ejemplo de } \\
\text { buenas estrategias y consciencia se pueden lograr cambios positivos que hagan del turismo una práctica divertida y } \\
\text { a la vez interesada en cuidar el planeta. } \\
\text { Los tipos de adaptación propuestos son: en la educación: impartir capacitaciones sobre el cuidado y protección de } \\
\text { flora y fauna; en la política; campañas sobre conservación de agua y aprovechamiento de tierras, campañas sobre el } \\
\text { peligro de la radiación ultravioleta (UV) y planes de gestión para evitar la construcción. }\end{array}$ \\
\hline
\end{tabular}


Tabla 2 Caracterizar desde la visión antropológica las comunidades La Curia, La Línea, La Cabuya y San Jacinto pertenecientes a la parroquia Pedro Pablo Gómez.

\begin{tabular}{|c|c|}
\hline \multicolumn{2}{|r|}{ La Curia } \\
\hline 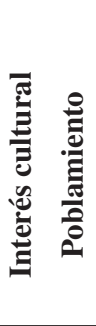 & $\begin{array}{l}\text { Según recuerdan los lugareños, los primeros pobladores son del mismo sitio. Los primeros habitantes tenían } \\
\text { como apellidos: Jaramillo Cruz y Cruz Rodríguez. } \\
\text { La motivación de vivir en este sitio, dicen los residentes, siempre fue la cosecha del café, ya que desde hace } \\
\text { muchos años era rentable. } \\
\text { La primera casa de La Curia, se ubica alrededor de la cancha. A lo largo de los años, la comunidad ha cambiado } \\
\text { mucho, ya que en los últimos años ha sido beneficiada con los servicios de luz y agua, y un camino regularmente } \\
\text { accesible que permite la entrada de los automóviles. }\end{array}$ \\
\hline 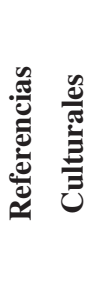 & $\begin{array}{l}\text { En la comunidad, existe la presencia de una capilla católica. Cuentan los residentes que aquí no hay historias } \\
\text { mitológicas o la existencia de algún ser sobrenatural. } \\
\text { La gastronomía de La Curia mantiene lo autóctono del cantón y de la provincia, teniendo como plato tradicional } \\
\text { el seco y el caldo de gallina criolla, las tortillas de maíz y el greñoso. } \\
\text { Las fiestas patronales de la comunidad rinden homenaje a la Virgen de Agua Santa, y se celebran del } 8 \text { al } 15 \text { de } \\
\text { septiembre. }\end{array}$ \\
\hline
\end{tabular}

\begin{tabular}{|l|l|}
\hline \multicolumn{1}{|c|}{ San Jacinto } \\
\hline & $\begin{array}{l}\text { Esta comunidad tiene alrededor de } 80 \text { familias, las cuales en su mayoría están compuestas por } 4 \text { o } 5 \text { personas. } \\
\text { Presentan un alto porcentaje de menores de edad. }\end{array}$ \\
& $\begin{array}{l}\text { Los habitantes de San Jacinto dicen que sus antepasados llegaron del cantón Paján, de la provincia de Manabí. } \\
\text { La primera familia que llegó a habitar esta comunidad fue la familia Cali. El motivo de lamigración a este sitio, } \\
\text { fue porque las tierras eran fértiles para los cultivos. Aquí ellos cultivan y comercializan la tagua, la cabuya y el } \\
\text { caucho. }\end{array}$ \\
& $\begin{array}{l}\text { La mayoría de las personas que hoy en día habitan la comunidad, han nacido ahí, y también han decidido formar } \\
\text { su familia en el mismo lugar. }\end{array}$ \\
\hline $\begin{array}{l}\text { En esta comunidad, existe la presencia de capillas y templos evangélicos, en los cuales los locales se dirigen a } \\
\text { orar con devoción. Todos los años celebran con euforia las fiestas patronales de la comunidad que son el 15 de } \\
\text { septiembre. }\end{array}$ \\
$\begin{array}{l}\text { Para esta comunidad uno de los acontecimientos más importantes ha sido la creación de la escuela de hace más } \\
\text { de } 60 \text { años. }\end{array}$ \\
$\begin{array}{l}\text { La presencia del cementerio es muy significativa, ya que es cercano y se encuentra dentro de la misma } \\
\text { comunidad, y no hay la necesidad de tener que salir fuera, con unos 10 años de creación. }\end{array}$
\end{tabular}

\begin{tabular}{|l|l|}
\hline \multicolumn{2}{|c|}{ La Cabuya } \\
\hline \multicolumn{2}{|c|}{$\begin{array}{l}\text { La comunidad La Cabuya cuenta con un aproximado de } 8 \text { familias, compuestas por } 4 \text { o } 5 \text { personas. Sus primeros } \\
\text { pobladores, según los moradores fueron hace } 80 \text { a } 100 \text { años provenientes de Paján; la familia Macías. Es } \\
\text { conocida así porque según la historia que han transmitido sus antepasados, en una montaña había un cabuyal y } \\
\text { desde entonces los habitantes llaman su comunidad de esta manera. La motivación de vivir en este sitio, dicen }\end{array}$} \\
\hline
\end{tabular}


Tabla 2 Caracterizar desde la visión antropológica las comunidades La Curia, La Línea, La Cabuya y San Jacinto pertenecientes a la parroquia Pedro Pablo Gómez.....(continuación)

\begin{tabular}{|c|c|}
\hline \multicolumn{2}{|r|}{ La Linea } \\
\hline 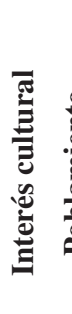 & $\begin{array}{l}\text { La comunidad La Línea cuenta con un aproximado de } 22 \text { familias, compuestas por } 4 \text { o } 5 \text { personas. Sus primeros } \\
\text { pobladores fueron hace } 70 \text { años provenientes de la comunidad aledaña San Jacinto; la familia Franco. La primera } \\
\text { casa que se construyó en la comunidad fue del presidente de la misma, acompañado con su familia El nombre de la } \\
\text { comunidad fue dado porque hace mucho tiempo había una carretera que era más corta para viajar hacia Colimes. } \\
\\
\text { La motivación para llegar a esta comunidad era el agradable contacto con la naturaleza, la tranquilidad, la paz y el } \\
\text { aprovechamiento de las tierras para los cultivos de café y maíz. Actualmente, los habitantes comentan que la comunidad } \\
\text { ha cambiado bastante, ya que en sus inicios no contaba ni con servicios básicos, ni tampoco con una carretera digna. Se } \\
\text { dedican a la agricultura hombres y mujeres, que es el principal sustento. }\end{array}$ \\
\hline 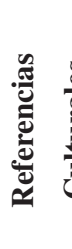 & $\begin{array}{l}\text { Desde hace mucho tiempo en la comunidad, celebran en la primera semana de septiembre sus fiestas patronales, donde } \\
\text { se realizan juegos dinámicos como el ensacado, el huevo en la cuchara, palo encebado, fútbol hombres y mujeres, a las } \\
\text { que acuden habitantes de comunidades vecinas. } \\
\text { Son muy devotos a la virgen de la Inmaculada Concepción. En esta comunidad, se pueden encontrar plantas medicinales } \\
\text { como son: la sábila, naranja agria, hierba luisa y existen curanderos ancestrales que ayudan a la comunidad. Tienen } \\
\text { leyendas e historias contadas por sus antepasados como la llorona y el duende. }\end{array}$ \\
\hline
\end{tabular}

Tabla 3 Analizar el comportamiento de la actividad de producción y distribución económica mediante gráficos y análisis estadísticos de las comunidades La Curia, La Línea, La Cabuya y San Jacinto pertenecientes a la parroquia Pedro Pablo Gómez.

\begin{tabular}{|c|c|}
\hline \multicolumn{2}{|r|}{ Actividad de producción y distribución económica } \\
\hline $\begin{array}{l}\text { Productos que siem- } \\
\text { bran en el sector }\end{array}$ & $\begin{array}{l}\text { El producto más cosechado en las comunidades es el maíz, este es el principal producto } \\
\text { que les genera ingresos económicos. Hay más productos como el café, el cacao, el limón, la } \\
\text { naranja y la yuca. Además, otros productos como pepino, naranjilla y demás frutas y verduras } \\
\text { que son usados para el consumo de las familias. }\end{array}$ \\
\hline Ingresos & $\begin{array}{l}\text { Los ingresos mensuales en las comunidades por lo general son de menos de } 200 \text { dólares } \\
\text { ya que solo se dedican a la agricultura y como mencionan no ganan mucho por la venta } \\
\text { de sus productos. Aunque en ciertas ocasiones cuando la cosecha es abundante y existen } \\
\text { compradores pueden ganar hasta un máximo de } 300 \text { dólares mensuales. }\end{array}$ \\
\hline $\begin{array}{l}\text { Venta de la produc- } \\
\text { ción }\end{array}$ & $\begin{array}{l}\text { La producción que se cosecha en las comunidades La Curia, San Jacinto, La Línea y la } \\
\text { Cabuya por lo general es vendida a minoristas que no les generan altos ingresos y muy rara } \\
\text { vez a mayoristas. Asimismo, los venden el mercado de la localidad y lo demás son para el } \\
\text { consumo de cada familia. }\end{array}$ \\
\hline $\begin{array}{l}\text { Mejora de la situa- } \\
\text { ción económica por } \\
\text { producción }\end{array}$ & $\begin{array}{l}\text { Un porcentaje considerable manifestó que su situación económica ha sido la misma por } \\
\text { algunos años y que solo las veces que existe abundante y buena cosecha aumentan un poco } \\
\text { más sus ingresos, pero por lo general se mantiene en el mismo porcentaje y como se muestra } \\
\text { anteriormente, sus ingresos son menores de } 200 \text { dólares. Otros expresaron que su situación } \\
\text { económica si ha mejorado gracias a la venta de sus productos principalmente por el maíz } \\
\text { amarillo. }\end{array}$ \\
\hline $\begin{array}{l}\text { La agricultura y su } \\
\text { aporte al desarrollo } \\
\text { comercial- } \\
\text { económico del } \\
\text { sector }\end{array}$ & $\begin{array}{l}\text { Desde sus inicios se han dedicado a la agricultura convirtiéndose en su principal actividad } \\
\text { que genera ingresos. Para los habitantes de las comunidades La Curia y San Jacinto están } \\
\text { conscientes de que últimamente esta actividad no está aportando mucho. Antes este si } \\
\text { generaba buenos ingresos, pero ahora lo básico para poder cubrir necesidades. Mientras en } \\
\text { las comunidades La Línea y La Cabuya, la mayoría expresó que sí ha aportado al desarrollo } \\
\text { de las familias y del sector. }\end{array}$ \\
\hline Otras actividades & $\begin{array}{l}\text { La mayoría de las personas en estas comunidades están dedicadas } 100 \% \text { a la agricultura } \\
\text { siendo su mayor sustento, además hay ciertas personas que si se dedican a la crianza y venta } \\
\text { de cerdos, aves, vacas y otros. También, manifestaron que hay tiempos en que van a trabajar } \\
\text { fuera como en albañilería o carpintería, pero que son por periodos de tiempo muy cortos. }\end{array}$ \\
\hline
\end{tabular}


Tabla 4 Identificar a los stakehol ders y su aporte en las comunidades La Curia, La Línea, La Cabuya y San Jacinto pertenecientes a la parroquia Pedro Pablo Gómez.

\begin{tabular}{|c|c|}
\hline \multicolumn{2}{|c|}{$\begin{array}{c}\text { Stakeholders y su aporte en las comunidades La Curia, La Línea, La Cabuya y San Jacinto } \\
\text { pertenecientes a la parroquia Pedro Pablo Gómez }\end{array}$} \\
\hline $\begin{array}{l}\text { Víctor Pita Poveda } \\
\text { presidente del GAD } \\
\text { de Pedro Pablo Gó- } \\
\text { mez }\end{array}$ & $\begin{array}{l}\text { Su labor principal es la administración y gobierno de la parroquia, incentivar actividades } \\
\text { de producción e integración y escescucharuchando las necesidades de las comunidades } \\
\text { que le pertenece. }\end{array}$ \\
\hline $\begin{array}{l}\text { Arturo Bailón presi- } \\
\text { dente de la comuni- } \\
\text { dad La Curia }\end{array}$ & $\begin{array}{l}\text { Encargado de organizar, integrar y escuchar las necesidades de la comunidad. Gestiona e } \\
\text { interviene para el bien y desarrollo de sus habitantes principalmente por el sector agrícola. } \\
\text { Su idea es implementar la modalidad de agroturismo; ya que cuentan con grandes } \\
\text { hectáreas de sembríos que podrían ser dadas a conocer a turistas en largas caminatas y } \\
\text { recorridos con el fin de aprender de dicha actividad agrícola. } \\
\text { Además, propone asociarse con la comunidad cercana Cristóbal Colón donde existen unas } \\
\text { cavernas que serían un gran atractivo para los turistas. }\end{array}$ \\
\hline $\begin{array}{l}\text { Víctor Franco presi- } \\
\text { dente de la comuni- } \\
\text { dad San Jacinto }\end{array}$ & $\begin{array}{l}\text { Encargado de organizar, integrar y escuchar las necesidades de su comunidad. Gestiona e } \\
\text { interviene para el bien y desarrollo de sus habitantes principalmente por el sector agrícola. } \\
\text { Su idea como todas las comunidades analizadas, es implementar el agroturismo ya que } \\
\text { esta es la actividad a lo que más se dedican y por ende tienen conocimiento. Propone crear } \\
\text { senderos para apreciar los sembríos con los que cuenta la comunidad. También le interesa } \\
\text { que se dé a conocer su gastronomía tradicional. }\end{array}$ \\
\hline $\begin{array}{l}\text { Esmer Baque Ma- } \\
\text { cías presidente de la } \\
\text { comunidad La Cabu- } \\
\text { ya }\end{array}$ & $\begin{array}{l}\text { Encargado de organizar, integrar y escuchar las necesidades de su comunidad. Gestiona e } \\
\text { interviene para el bien y desarrollo de sus habitantes principalmente por el sector agrícola. } \\
\text { Como presidente y en representación de la comunidad, ve rentable el agroturismo para } \\
\text { hacer conocer la labor que enfrenta día a día el agricultor. Quisiera implementar senderos, } \\
\text { ya que existe vegetación interesante y poder practicar ciclismo para mantener contacto } \\
\text { con la naturaleza. Resaltan también, una hacienda que se podría adecuar para ofrecer } \\
\text { hospedaje para quienes se interesen por el agroturismo. }\end{array}$ \\
\hline $\begin{array}{l}\text { Ramón Franco pre- } \\
\text { sidente de la comuni- } \\
\text { dad La Línea }\end{array}$ & $\begin{array}{l}\text { Encargado de organizar, integrar y escuchar las necesidades de su comunidad. Gestiona e } \\
\text { interviene para el bien y desarrollo de sus habitantes principalmente por el sector agrícola. } \\
\text { Su principal idea son las cabañas y un parque ecológico, pues hay una elevación que se } \\
\text { podría aprovechar para senderismo, ciclismo y avistamiento de flora y fauna. Y como las } \\
\text { demás comunidades, el agroturismo con sus respectivos guías. }\end{array}$ \\
\hline
\end{tabular}

\section{Conclusiones}

Las comunidades La Curia, San Jacinto, La Línea y La Cabuya mantienen una biodiversidad faunística y florística similar. Estas han mantenido su hábitat natural gracias a que no existe aún mucha contaminación en su entorno, además, en estas comunidades no existe desarrollo turístico, ni una infraestructura que impacte desde los puntos positivos o negativos al medio ambiente.

Las ya mencionadas comunidades, han tenido cambios desde la llegada de sus primeros pobladores que fueron atraídos por las fértiles tierras para la cosecha del café en el sector. En la actualidad, los pobladores mantienen su identidad cultural celebrando sus fiestas más representativas, preparando deliciosos platillos manteniendo el sabor y técnicas de sus antepasados.

Las cuatro comunidades se dedican el $100 \%$ a la agricultura, siendo su principal sustento económico, la misma que cuenta con tiempos de alta cosecha en las que se puede generar un poco más de ingresos y la baja cosecha en la que la producción es escasa. Sus productos son por lo general vendidos a minoristas y usados para el consumo de cada una de las familias. 
Los stakeholders, son parte importante de cada comunidad tratando siempre de contribuir para el desarrollo de cada una de ellas. Sus presidentes son los agentes involucrados en la necesidad de incentivar, solicitar mejoras y buscar soluciones a los problemas que se generen.

\section{Recomendaciones}

Es importante que se siga manteniendo y conservando el entorno natural que rodea estas comunidades. De la misma forma sería una buena estrategia que poco a poco se vayan implementando modalidades turísticas que aporten al desarrollo de las comunidades siempre y cuando sea de una manera sostenible para que se logre un impacto positivo.

Como comunidades es de suma relevancia que sigan vivas las raíces y se practiquen tradiciones con el fin de mantener la cultura. Sería necesario que sus fiestas sean celebradas con todo el fulgor que lo amerita, recordando que es el sello que los caracteriza y que los mismos moradores vayan impartiendo a cada uno que llega, las riquezas culturales que son parte de la historia.

La producción es clave para el desarrollo de las comunidades, por lo que con una buena gestión se podría llegar al mercado nacional y de esta forma los habitantes tengan mejores ingresos. Para ellos, es una clave que los capaciten en la actividad agrícola para que pueda ser aprovechada al máximo y en el ámbito turístico para generan futuras entradas económicas.
Como agentes que intervienen en las 4 comunidades, es necesario que mantengan una buena relación con sus moradores y que a través de nuevas ideas se busque implementar el turismo como otra fuente de ingreso.

\section{Referencias Bibliográficas}

Briones, G. (s.f.). Metodologia de la investigacion. Bogota.

Freeman. (1984). Strategic management, a stakeholder approach.

Jara, O. (2014). La sistematización de experiencias, practica y teorica para otros mundos posibles. Lima.

Recibido: 15 de mayo de 2019

Aceptado: 3 de julio de 2019 